\title{
The flow and flow-induced noise behaviour of a simplified high-speed train bogie in a cavity and including a fairing
}

\author{
J. Y. Zhu ${ }^{1,3}$, Z. W. Hu', D. J. Thompson²
}

\begin{abstract}
${ }^{1}$ Aerodynamics and Flight Mechanics Research Group, Faculty of Engineering and the Environment, University of Southampton, Southampton SO17 1BJ, United Kingdom

2 Institute of Sound and Vibration Research, Faculty of Engineering and the Environment, University of Southampton, Southampton SO17 1BJ, United Kingdom

${ }^{3}$ Institute of Railway and Urban Mass Transit, Tongji University, Shanghai 200092, People's Republic of China
\end{abstract}

\begin{abstract}
Aerodynamic noise is a significant source for high-speed trains but its prediction in an industrial context is difficult to achieve. The flow and aerodynamic noise behaviour of a simplified high-speed train bogie at scale 1:10 are studied here through numerical simulations. The bogie is situated in a cavity beneath the train and the influence of a bogie fairing on the flow and flowinduced noise developed around the bogie area is investigated. A two-stage hybrid method is used, combining computational fluid dynamics and an acoustic analogy. The near-field unsteady flow is obtained by solving the unsteady three-dimensional Navier-Stokes equations numerically using delayed detached-eddy simulation and the data are utilized to predict the far-field noise based on the Ffowcs Williams-Hawkings acoustic analogy. Results show that when the bogie is located inside the bogie cavity, the shear layer developed from the cavity leading edge interacts strongly with the flow separated from the bogie upstream components and the cavity walls. Therefore, a highly turbulent flow is generated within the bogie cavity due to the strong flow impingements and flow recirculations occurring there. For the case without the fairing, the surface shape discontinuity in the bogie cavity along the carbody side walls generates strong flow unsteadiness around these regions. When the fairing is mounted in front of the bogie cavity, the flow interactions between the bogie cavity and the outer region are reduced and the development of turbulence outside the fairing is greatly weakened. Based on predictions of the noise radiated to the trackside using a permeable data surface parallel to the carbody side wall, it is found that the bogie fairing is effective in reducing the noise generated in most of the frequency range and a noise reduction around $5 \mathrm{~dB}$ is achieved in the farfield for the current model case.
\end{abstract}

\section{Introduction}

Aerodynamic noise becomes significant for high-speed trains at running speeds over about $300 \mathrm{~km} / \mathrm{h}$ [1-3]. The main aeroacoustic sources on high-speed trains identified by different studies are the bogie, the pantograph, the recess of the pantograph, the inter-

E-mail addresses:

zhujianyue@tongji.edu.cn (J. Y. Zhu), z.hu@soton.ac.uk (Z.W. Hu), djt@isvr.soton.ac.uk (D. J. Thompson) 
coach spacing, etc $[2,4]$. As an example, the Korean high-speed train pass-by noise was measured at a speed of $300 \mathrm{~km} / \mathrm{h}$ using the microphone arrays [5]. Based on the measured noise map, the bogies, pantographs and inter-coach gaps were found to be the main noise sources. As one of the main aerodynamic noise sources of high-speed trains, the bogie is a complex structure containing many components with surface shape variations and discontinuities, and thus the flow generated around it is highly turbulent $[2,3]$. In terms of the noise contribution to the overall noise of the whole train, the aerodynamic noise produced from the bogie areas has been estimated to be around $15 \mathrm{~dB}$ larger than that from the pantograph [2].

Most aeroacoustic studies on the bogie area have been performed through anechoic wind tunnel experiments and field tests. Noise radiated from a 1:7 scale simplified bogie located in the bogie cavity of a train model was measured in [6]. The results showed that rounding the downstream edge of the bogie cavity and the use of side skirts reduced the noise. The main noise contributions came from the bogie components displaced outwards into the flow. The differences in the inflow speeds at the bogie cavity along the train produced the different noise generation. In [7] the identification of bogie aerodynamic sources was investigated with on-board measurements using the coherent output power technique. It was shown that the coherent source mechanisms from the bogie area were complex and the bogie should be considered as several uncorrelated acoustic sources.

Recently, the aerodynamic and aeroacoustic behaviour of the flow past an isolated wheelset [8] and an isolated simplified bogie [9] was studied numerically and it was found that surface pressure fluctuation and vortex shedding around the geometries were the key factors for the aerodynamic noise generation. The directivity exhibits a dipole-like shape for the noise radiated from the two cases. Note that the flow and flow-induced noise behaviour of the bogie inside the bogie cavity will be different from the isolated bogie case.

It has been shown that by adding the fairings around the bogie areas to modify the flow developed there, the drag and aerodynamic noise of high-speed trains can be reduced [10]. Flush-mounted fairings were fitted on the leading bogie of the Series 700 Shinkansen and aerodynamic noise reductions were obtained by improving the flow 
passing over the bogie regions and by shielding the aerodynamic noise sources [11]. Compared with these studies based on the experimental measurements, this paper aims to study numerically the influence of a bogie fairing on the aerodynamic and aeroacoustic behaviour of the flow passing over a simplified bogie located inside the bogie cavity. The details of the flow behaviour and the corresponding aeroacoustic mechanisms from the bogie region will be investigated.

For practical reasons the geometry is simplified and is studied at 1:10 scale. Moreover, a reduced flow speed of $30 \mathrm{~m} / \mathrm{s}$ is considered. Nevertheless, the main features of the flow field are expected to be similar to those occurring at full scale. The main difference will be in the frequency content: for $1: 10$ scale and approximately $1 / 3$ of the full-scale speed the frequency content will be shifted upwards by a factor of around 3 . Wheel rotation is neglected but in previous simulations for an isolated wheelset this has been found to have little effect on the sound generation $[8,12]$.

\section{Numerical Method and Simulation Setup}

Numerical simulations are carried out using a two-stage strategy of computational fluid dynamics and computational aeroacoustics methods. Aerodynamically, high-speed trains are operating within the low Mach number flow regime, up to about 0.2-0.3. The incoming flow simulated here is at an even lower Mach number of 0.09 (corresponding to $30 \mathrm{~m} / \mathrm{s}$ ). Therefore, the compressibility effects may be neglected in studying the hydrodynamics of the flow field. Moreover, at low Mach number the dominant noise sources are the dipole sources generated from wall pressure fluctuations, which can be predicted essentially through incompressible flow modelling [13]. For an open cavity case at a Mach number of 0.15 as an example, Ask and Davidson [14] found that the sound source intensity on the cavity wall matched well between the incompressible and compressible flow solutions which were applied as the noise sources to the acoustic analogy method. Thus, the unsteady, incompressible Navier-Stokes equations are used here to solve the flow field. The open source software OpenFOAM-2.2.2 is employed for flow simulations. A scheme of second-order accuracy is used for the spatial derivatives and the temporal discretization follows a second-order fully implicit scheme. The delayed detached-eddy simulation (DDES) based on the Spalart-Allmaras (S-A) turbulence model is applied for 
the flow calculations [15]. The near-field unsteady flow computation provides acoustic sources, which are applied in the Ffowcs Williams-Hawkings (FW-H) acoustic analogy for far-field noise predictions [16-18].

As in [9], a simplified bogie is considered at scale 1:10. This, combined with the reduced flow speed of $30 \mathrm{~m} / \mathrm{s}$, is chosen to prevent the computational mesh required from becoming excessively large. Fig. 1 shows the models of the simplified bogie at 1:10 scale situated inside the bogie cavity without and with the fairing used in this study. The bogie centre is located at $x=y=z=0$. The axle has a diameter $(d)$ of $17.5 \mathrm{~mm}$ and the wheels have a diameter $(D)$ of $92 \mathrm{~mm}$. The wheelbase (centre-to-centre distance between the two axles) is $252 \mathrm{~mm}$ which is about 14 times the axle diameter. The carbody under-floor surfaces are $4 \mathrm{~mm}$ above the bogie horizontal central plane. The half width of the carbody is $130 \mathrm{~mm}$. The height of the bogie cavity is $70 \mathrm{~mm}$. The distance from the cavity leading edge to the centreline of the first axle is $76 \mathrm{~mm}$. The distance from the outside of the bogie frame to the inside of the fairing is $12 \mathrm{~mm}$. The fairing is shown in Fig. 1(b). It has a thickness of $3.5 \mathrm{~mm}$ and an identical length and depth to the bogie cavity. The top half of the bogie is thus situated within the bogie cavity and covered by the fairing. There is no ground in the model and the wheels are not rotating.

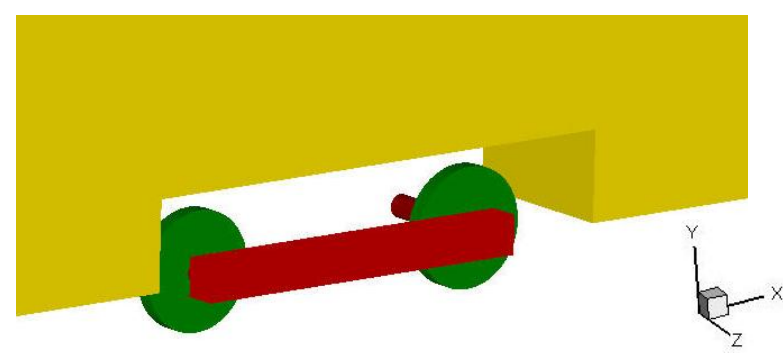

(a) Without fairing

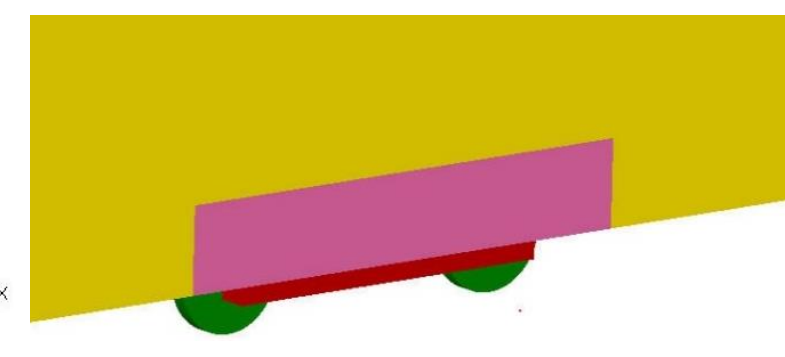

(b) With fairing

Fig. 1. Models of a simplified bogie inside the bogie cavity (1:10 scale)

The bogie and cavity are assumed to be symmetrical about the vehicle mid-plane where the influence of the three-dimensional flow from the wheel and frame is small. Therefore, it is reasonable to include only half of the geometry and make use of the symmetry to reduce the computational cost. The computational domain has overall dimensions $20.7 D$, $11 D$ and $6.3 D$ (where $D=92 \mathrm{~mm}$ is the wheel diameter) along the streamwise $(x)$, vertical $(y)$ and spanwise ( $z$ ) directions, respectively. Thus, the outlet and side boundaries are far 
enough away to have negligible influence on the flow developed around the bogie and the cavity.

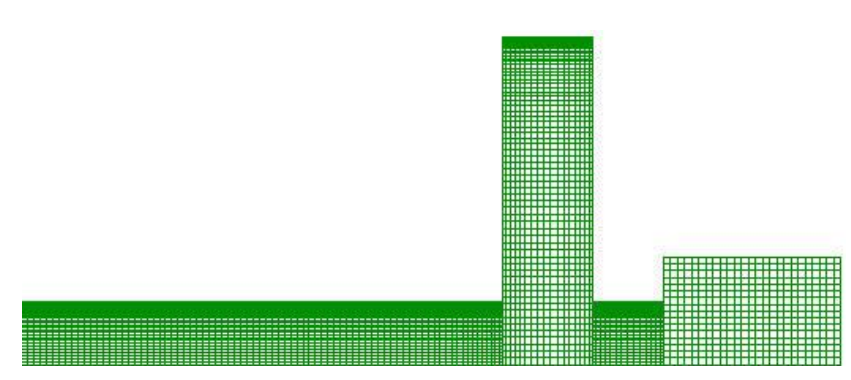

(a) Bogie surfaces

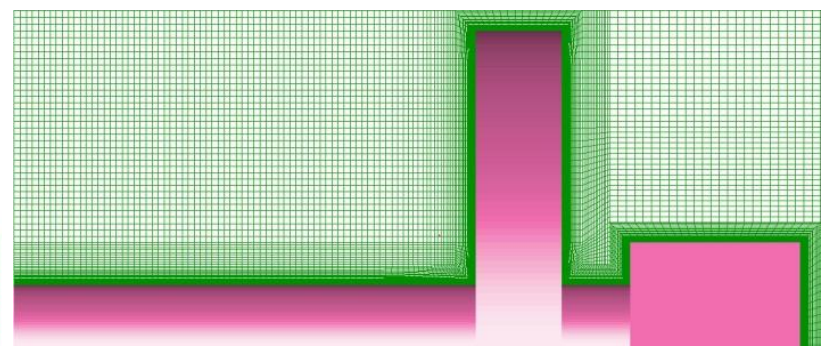

(b) Wheelset vertical mid-plane

Fig. 2. Structured mesh topology around the bogie

A rigorous grid convergence study for a complex geometry case is difficult to achieve because of the large calculations required for the unsteady flow. As a main part of the wheelset, the axle is a typical circular cylinder. Based on the mesh refinement study for the cylinder case $[9,12]$, a fully structured mesh is generated around all geometries with resolutions similar to the 'Baseline' grid of the cylinder case. The cell size on the axle surface is implemented as $0.42 \mathrm{~mm}$ around the perimeter and $0.88 \mathrm{~mm}$ in the spanwise direction. The maximum cell size on the wheel, frame, fairing and carbody surface is up to $1 \mathrm{~mm}$. The mesh in the corner area between the wheel and axle is refined with double grid points in the wheel radial direction and the axial direction of the axle. As an example, the mesh around the bogie is displayed in Fig. 2. The distance from the bogie, carbody wall and fairing surface to the nearest grid point is set as $1 \times 10^{-5} \mathrm{~m}$ and stretched with a growth ratio of 1.1 inside the boundary layer. This yields a maximum value of $y^{+}$(the dimensionless first-cell spacing, $y^{+}=\frac{y u_{\tau}}{v}$, where $y$ is the distance from the wall, $u_{\tau}$ the friction velocity and $v$ kinetic viscosity) less than 1 for all cases, which ensures that the boundary layer is resolved properly and the turbulence model employed can account for the low-Reynolds number effects inside the viscous sublayer. This grid generation strategy results in a fully multi-block structured mesh in the entire domain with a total number of grid points of 36.5 million for the case without the fairing and 38.9 million for the case with the fairing. A similar mesh topology has previously been employed for the isolated wheelset and bogie cases in which good agreements were achieved between numerical simulations and experimental measurements for the radiated far-field noise $[8,9,12]$. 
The boundary conditions applied are as follows: the upstream inlet flow is represented as a steady uniform flow $\left(U_{\infty}=30 \mathrm{~m} / \mathrm{s}\right)$ with a low turbulence intensity. The top, bottom, axle mid-plane and side boundaries are specified as having symmetry boundary conditions which are equivalent to zero-shear slip walls; a pressure outlet with zero gauge pressure is imposed at the downstream exit boundary and all solid surfaces are defined as stationary no-slip walls. Simulations are run with a physical timestep size of $5 \times 10^{-6} \mathrm{~s}$ initially, increasing to $1 \times 10^{-5} \mathrm{~s}$, which gives an adequate temporal resolution for the implicit time marching scheme used. The Courant-Friedrichs-Lewy (CFL) number is less than 1 within most of the computational domain and has a maximum value of 2 . Taking the case of bogie-inside-cavity with fairing as an example, the calculations were parallelized over 320 processors on the Iridis4 cluster of the University of Southampton. Approximately 42,000 timesteps were required to reach a fully developed flow field and the collection of acoustic data were run further for around 66,000 timesteps to obtain a reasonable frequency resolution in data processing. The total time needed for the simulation was around 8 weeks. It would be very challenging to run multiple cases with different inflow and other conditions. Therefore, this contribution focuses on effects of the bogie fairing to flow aerodynamics and flow-induced noise at an incoming flow speed of $30 \mathrm{~m} / \mathrm{s}$.

\section{Aerodynamic Results}

In order to investigate the flow-field characteristics developed around the bogie, the calculation results of the instantaneous iso-surfaces of $Q$-criterion and the vorticity fields are displayed to get an overview of the unsteady flow; then, the simulation results of the turbulent kinetic energy, the fluctuating force coefficients and the bogie wall pressure fluctuations are compared and analyzed for the two cases, with and without the fairing.

\subsection{Flow field}

For the case with the fairing Fig. 3 visualises the vortical structures of the turbulent flow around the bogie and cavity. These are represented by the iso-surfaces of the second invariant of the velocity gradient $Q$, plotted at a normalised value of 50 (based on 
$Q /\left[\left(U_{\infty} / D\right)^{2}\right]$, where $U_{\infty}$ is the freestream velocity and $D$ is the wheel diameter). They are coloured by the non-dimensional velocity magnitude $\left(|U| / U_{\infty}\right)$. Fig. $3(\mathrm{a})$ shows that the vortices shed from the upstream components impinge on the downstream ones, generating a highly turbulent wake within and behind the bogie. Subsequently, all vortices impinge on the cavity rear wall, deform considerably and are merged into the eddies formed downstream behind the cavity. It can be observed from Fig. 3(b) that different scales of vortices are generated between the upstream wheelset and cavity top wall as the various flow interactions and flow impingements occur there. Since, in the region of the downstream half of the cavity and the cavity rear wall, the flow development is impeded by the fairing, the unsteady turbulent flow is reduced markedly in these areas compared with the case without the fairing, as shown in Fig. 4.

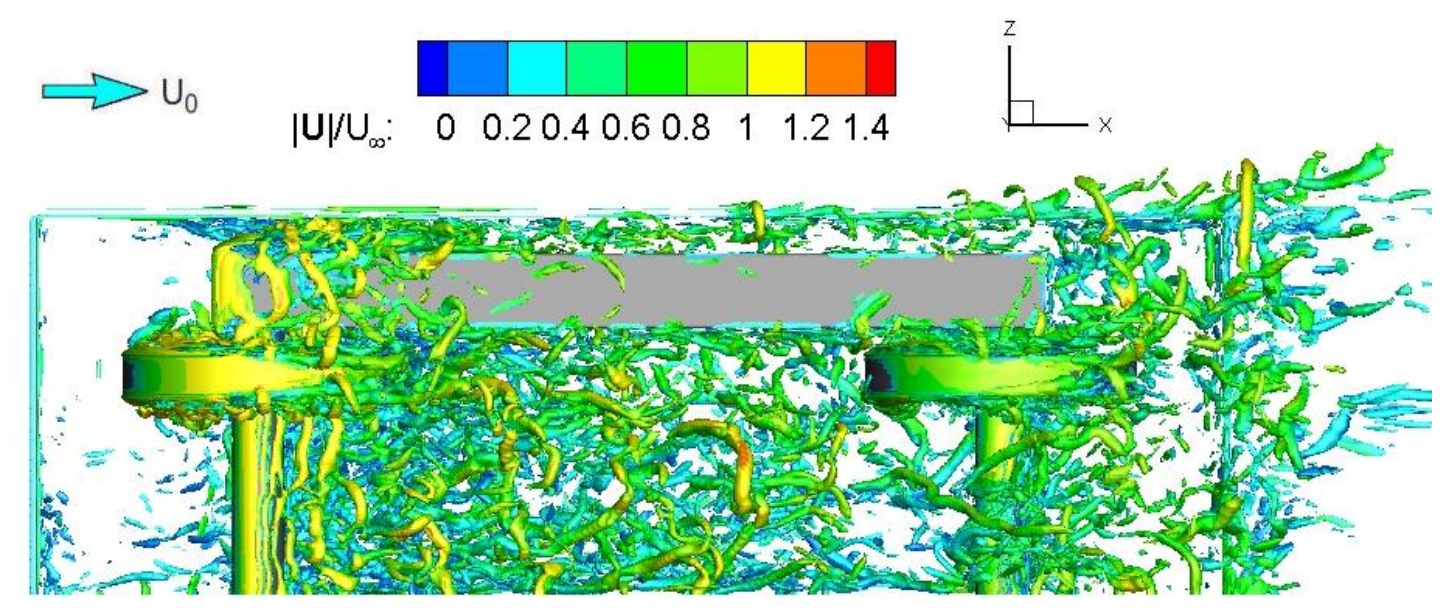

(a) Bottom view

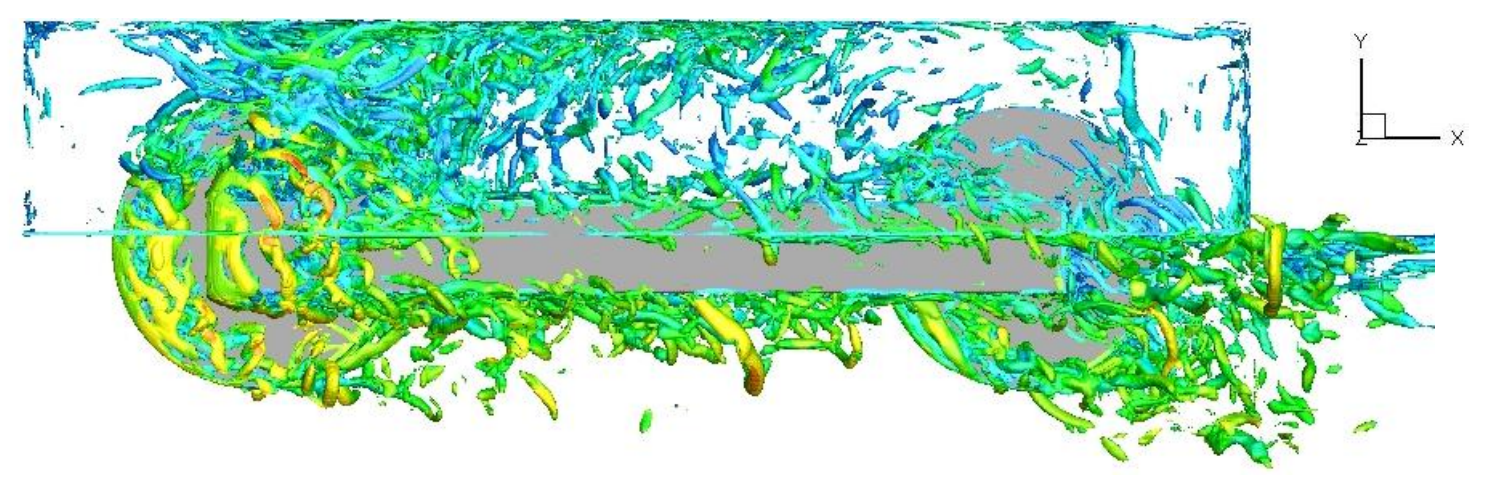

(b) Side view

Fig. 3. Iso-surfaces of the instantaneous normalised $Q$-criterion (case with the fairing) 


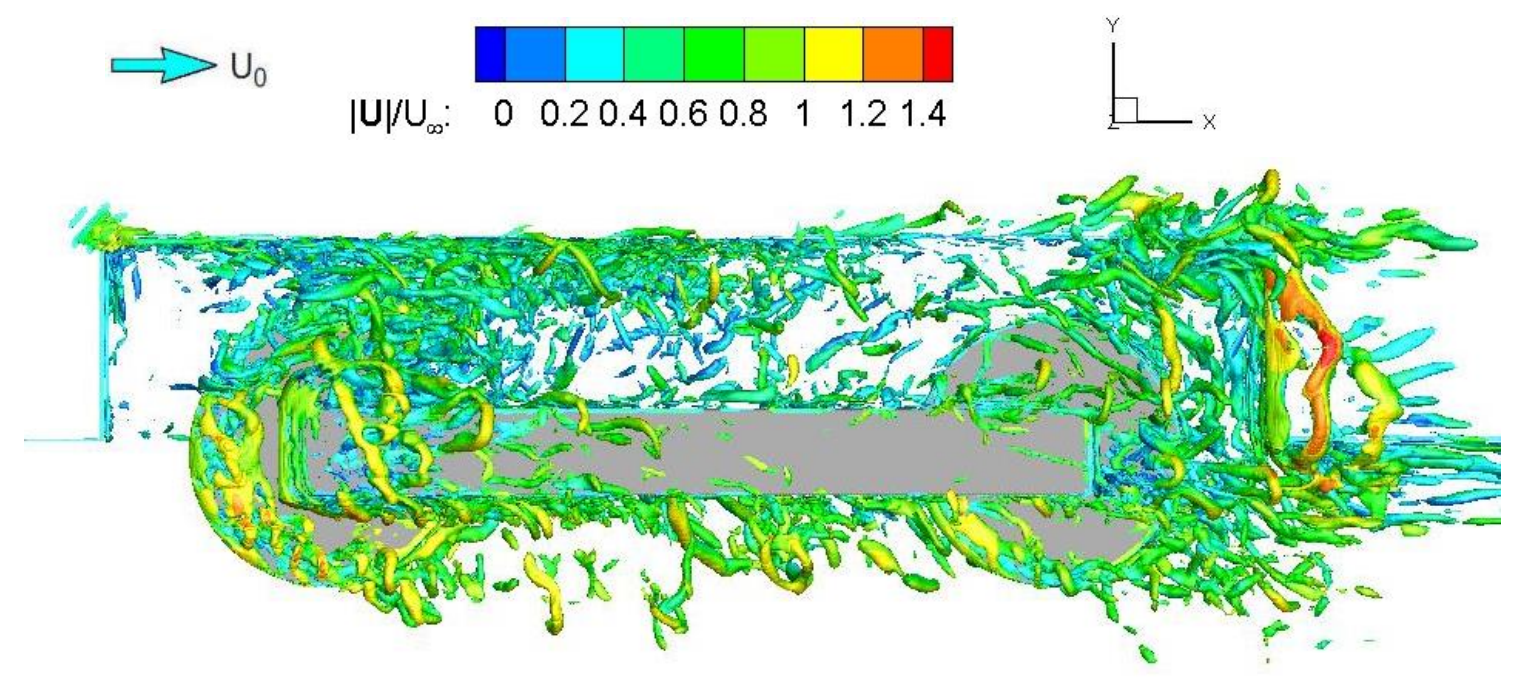

Fig. 4. Iso-surfaces of the instantaneous normalised $Q$-criterion (case without the fairing, side view)

Fig. 5 depicts the instantaneous non-dimensional spanwise vorticity field ( $\omega_{z}=$ $(\partial V / \partial x-\partial U / \partial y) D / U_{\infty}$, where $D$ is the wheel diameter) for the case with the fairing. These results are similar in the case without the fairing. Fig. 5(a) displays the instantaneous spanwise vorticity field contours in the plane midway between the wheel inner surface and the axle centre. This shows that a shear layer is shed from the cavity leading edge and bent upwards rapidly in the streamwise direction. This shear layer travels downstream and has a strong interaction with the flow separated from the upstream axle. Then, all vortices are mixed up and impinge on the cavity top wall, leading to an unsteady flow with complex structure being formed there. Additionally, it can be seen that the wake behind both the upstream and downstream axles is highly unsteady and developed mainly below the bogie cavity owing to the shielding effect from the fairing. Moreover, the vortices generated behind the rear axle impinge on the cavity rear corners, resulting in a highly irregular and unsteady wake there. 


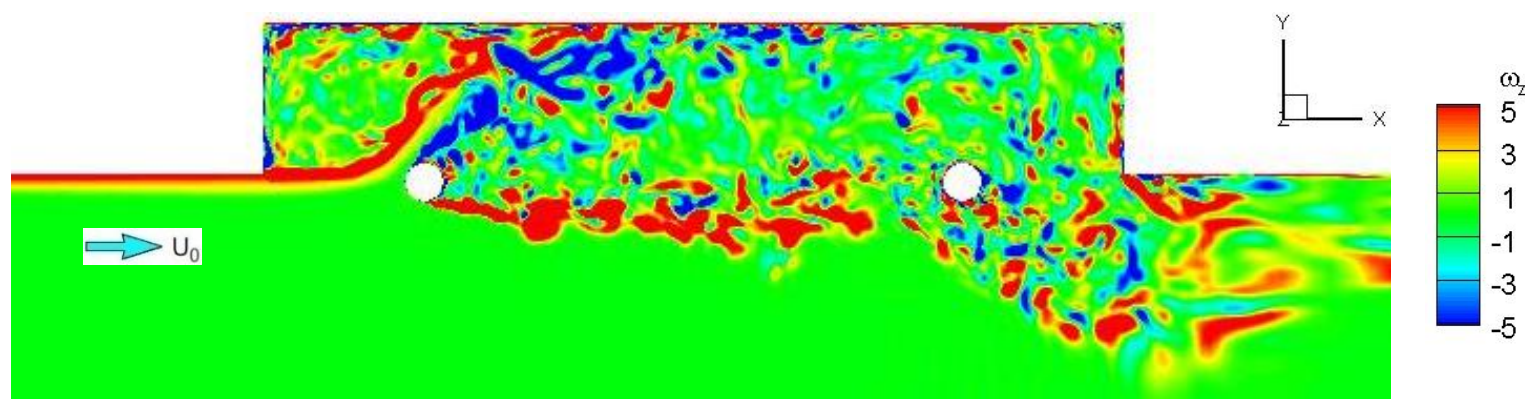

(a) Midway long half-axle

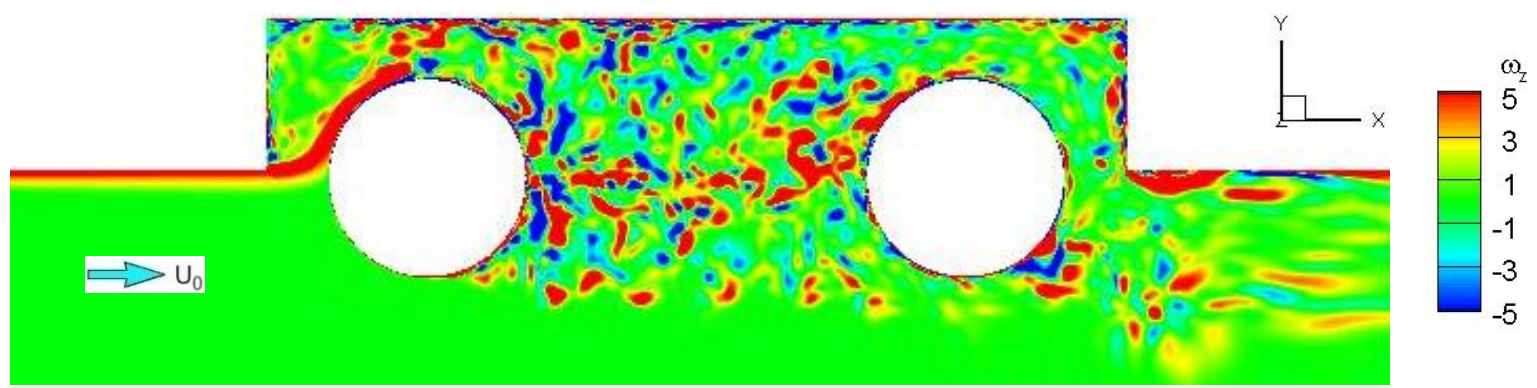

(b) Wheel mid-span

Fig. 5. Contours of the instantaneous spanwise vorticity field in a vertical plane for case with fairing (side views)

Fig. 5(b) shows the instantaneous spanwise vorticity contours in the wheel mid-span plane. The shear layer generated from the cavity leading edge is bent upwards and attached on the wheel tread as the gap between the wheel and cavity leading edge is relatively small; then, the vortices are separated from the wheel surfaces, leading to an unsteady wake forming behind the upstream wheel. Subsequently, the vortices are convected and impinge on the downstream wheel; however, the wake is relatively weak around the region between the downstream wheel and the cavity rear wall since the flow cannot become fully developed due to the shielding effect from the fairing.

The contours of the non-dimensional mean turbulent kinetic energy in a horizontal plane cut at $y=0.06 \mathrm{~m}$ (as indicated by the straight line in the sketch) through the bogie cavity are presented in Fig. 6 as a bottom view for the two cases. The non-dimensional mean turbulent kinetic energy is given by $k=\frac{1}{2}\left(\overline{u^{\prime} u^{\prime}}+\overline{v^{\prime} v^{\prime}}+\overline{w^{\prime} w^{\prime}}\right) / U_{\infty}{ }^{2}$, where $u^{\prime}, v^{\prime}$ and $w^{\prime}$ are velocity fluctuation components in $x, y$ and $z$ directions respectively and the overbar denotes a time average. The results are shown with similar scales and contour levels. It can be seen from Fig. 6(a) that for the case without the fairing, a high-level turbulence is also produced around the area of the bogie cavity rear wall corners due to the large 
turbulent flow impingements occurring there, and consequently a strong flow-induced noise will potentially be generated in this region. By comparison, for the case with the fairing shown in Fig. 6(b), most of the flow-field unsteadiness at a high level is generated in the wake region around the top area of the upstream wheelset as a result of the various flow interactions developed in this region.

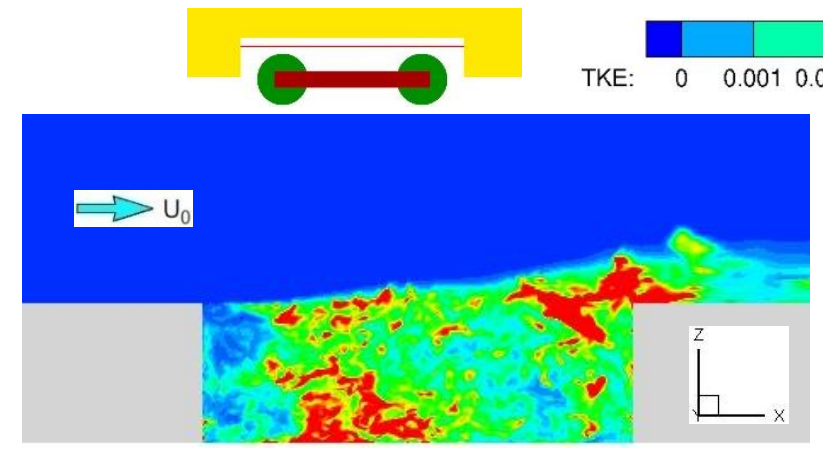

(a) Without fairing
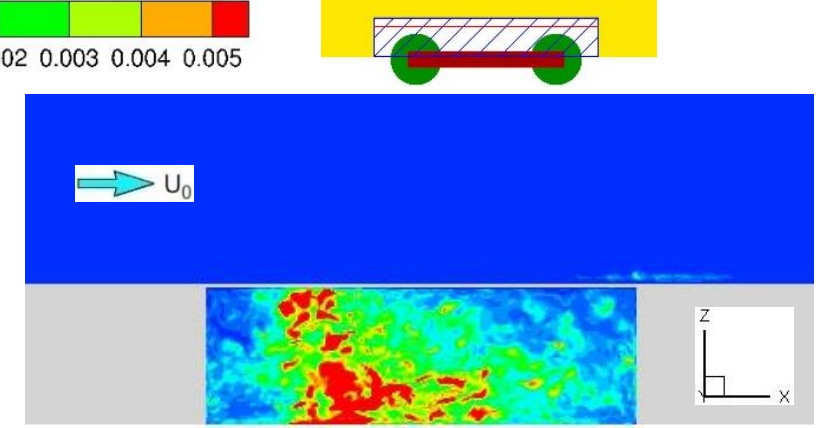

(b) With fairing

Fig. 6. Contours of the non-dimensional mean turbulent kinetic energy in a horizontal plane cut at $y=0.06 \mathrm{~m}$ through the bogie cavity above the wheels (bottom views)

\subsection{Fluctuating force coefficients}

Fig. 7 compares the power spectral densities (PSDs) of fluctuating lift, drag and side force coefficients of the half bogie for the cases with and without the fairing. These are defined as the fluctuating lift, drag and side forces on the bogie surfaces, non-dimensionalized by $\left(\frac{1}{2} \rho_{0} U_{\infty}^{2} A\right)$, where $A$ is the projected frontal cross-section area of the bogie. Fig. 7 shows that the lift and side force coefficients are similar for the cases with and without the fairing within the frequency range $200-2000 \mathrm{~Hz}$ and the drag coefficients are similar in most of the frequency range, indicating that the flow developed around the bogie in the two cases contains a similar turbulence structure with various scales. In the frequency range below $200 \mathrm{~Hz}$, the lift coefficient is higher for the case with the fairing (Fig. 7a). In contrast, a higher spectrum level can be seen between $40 \mathrm{~Hz}$ and $130 \mathrm{~Hz}$ in the side force coefficient of the case without the fairing (Fig. 7c), which may correspond to the interaction between the shear layer shed from the cavity side leading edge and the flow developed around the bogie. Note that at higher frequencies, above $2 \mathrm{kHz}$, the spectrum amplitude is comparatively larger in lift and side force coefficients for the case with the fairing, since more small-scale eddies are generated around the bogie in the cavity covered by the fairing. 


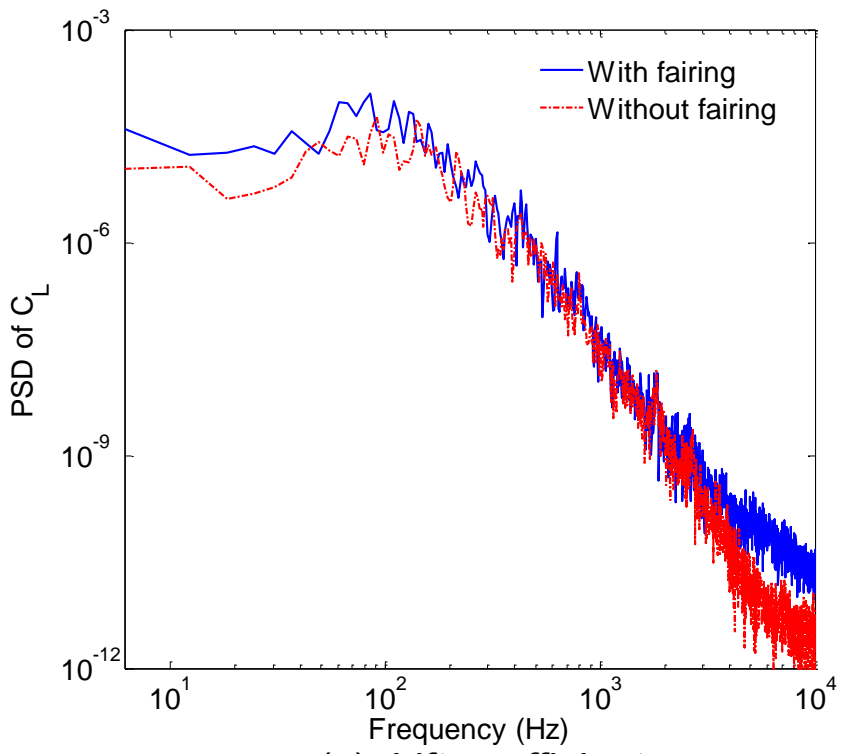

(a) Lift coefficient

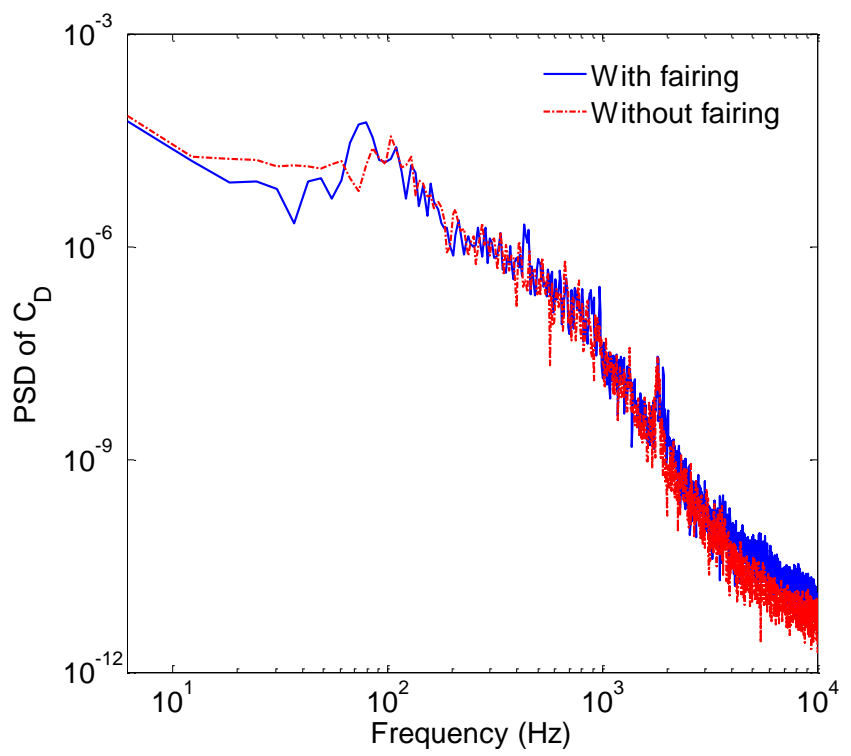

(b) Drag coefficient

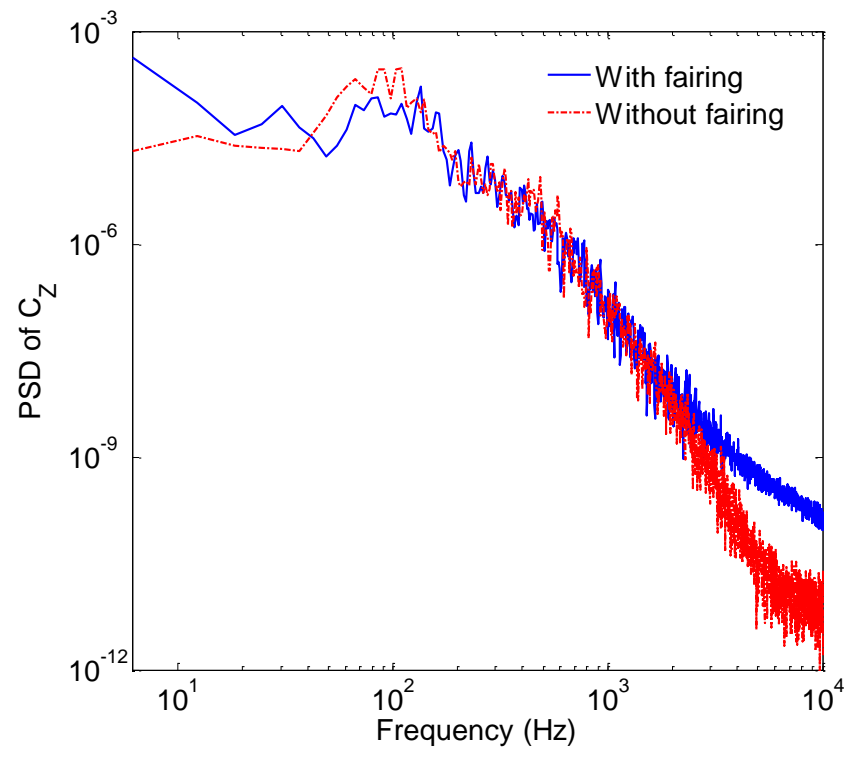

(c) Side force coefficient

Fig. 7. Power spectral densities of force coefficients of the bogie inside the cavity

\subsection{Wall pressure fluctuations}

Fig. 8 displays the wall fluctuating pressure level in decibels on the bogie surfaces for the cases without and with the fairing. This is given by $L_{p}=10 \log \left(\overline{p^{\prime 2}} / p_{\text {ref }}^{2}\right)$, where $\overline{p^{\prime 2}}$ is the mean-square fluctuating pressure and $p_{\text {ref }}$ is reference acoustic pressure $20 \mu P a$. This can be used to identify the potentially significant noise source regions. It shows that for both cases, a high pressure fluctuation appears on the wheel and frame inner side 
surface and the axle surface near the axle-wheel junction region; by comparison, the surface pressure fluctuations are considerably higher on these areas for the case with the fairing. This is due to the stronger flow interaction occurring around the bogie inside the cavity covered by the fairing. Moreover, similar distributions of wall pressure fluctuations to the ones in the figure are developed on the outer surfaces of the wheel and frame for both cases.

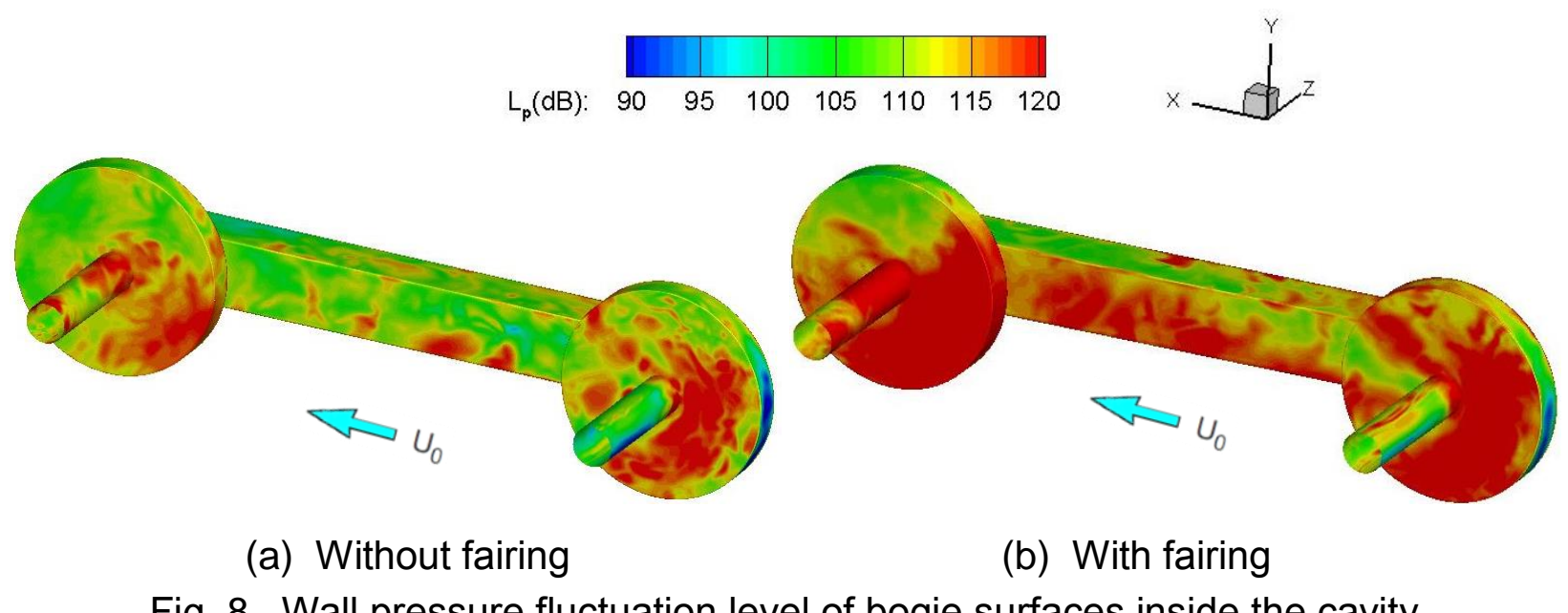

Fig. 8. Wall pressure fluctuation level of bogie surfaces inside the cavity

\section{Numerical Algorithm for Noise Prediction}

The classical FW-H equation assumes the propagation of sound waves in a quiescent medium and does not take into account the presence of a mean flow as occurs for windtunnel cases [16-18]. Thus, in the application of these formulations, the wind-tunnel problem should be transformed into a moving-observer problem where the observer is assumed to be moving at a constant speed in an environment at rest. If the observer remains stationary in the simulation of a wind-tunnel case, the moving mean flow should be taken into account in the computation. In order to calculate more efficiently the farfield noise generated from the two large cases of the bogie in the cavity, an aerodynamic noise prediction code using the moving-medium formulation has been implemented and validated by a number of test cases. 


\subsection{Numerical implementation}

Based on reference [19], the convective $\mathrm{FW}-\mathrm{H}$ equation for a wind-tunnel problem is presented in the following to understand the meaning of all terms in the formulations. Considering a mean flow moving with speed $\mathbf{U}_{0}$, the inhomogeneous convective $\mathrm{FW}-\mathrm{H}$ equation [19] is obtained as

$$
\begin{gathered}
{\left[\frac{\partial^{2}}{\partial t^{2}}+2 U_{0 i} \frac{\partial^{2}}{\partial t \partial x_{i}}-c_{0}^{2} \frac{\partial^{2}}{\partial x_{i} \partial x_{i}}+U_{0 i} U_{0 j} \frac{\partial^{2}}{\partial x_{i} \partial x_{j}}\right]\left[H(f) \rho^{\prime}\right]} \\
=\left(\frac{\partial}{\partial t}+U_{0 j} \frac{\partial}{\partial x_{j}}\right)\left[Q_{i} n_{i} \delta(f)\right]-\frac{\partial}{\partial x_{i}}\left[L_{i j} n_{j} \delta(f)\right]+\frac{\partial^{2}}{\partial x_{i} \partial x_{j}}\left[H(f) T_{i j}\right],
\end{gathered}
$$

where $\delta(f)$ is the Dirac delta function, $H(f)$ the Heaviside function $(H(f)=1$ for $f>0$ and $H(f)=0$ for $f<0)$ and $f(\mathbf{x}, t)=0$ represents the surfaces; $n_{i}$ corresponds to the local normal vector components; $c_{0}, \rho_{0}$ and $p_{0}$ are the speed of sound, density and pressure in the undisturbed free-stream medium, respectively. $\rho^{\prime}=\rho-\rho_{0}$ is the density fluctuation. The source terms are defined as

$$
\begin{gathered}
Q_{i}=\left[\rho\left(u_{i}-v_{i}+U_{0 i}\right)+\rho_{0}\left(v_{i}-U_{0 i}\right)\right], \\
L_{i j}=\rho u_{i}\left(u_{j}+U_{0 j}-v_{j}\right)+\left(p-p_{0}\right) \delta_{i j}-\tau_{i j}, \\
T_{i j}=\rho u_{i} u_{j}+\left[\left(p-p_{0}\right)-c_{0}^{2}\left(\rho-\rho_{0}\right)\right] \delta_{i j}-\tau_{i j},
\end{gathered}
$$

in which $Q_{i}$ and $L_{i j}$ are thickness and loading noise terms; $T_{i j}$ is the Lighthill stress tensor and the corresponding quadrupole contributions can be neglected for the current cases at low Mach numbers $[13,18]$. $\rho$ is the density and $p$ the pressure. $u_{i}$ and $v_{i}$ are the flow and surface velocity components in the $i$ th direction. $\tau_{i j}$ is the viscous stress tensor. $\delta_{i j}$ is the Kronecker delta, $\delta_{i j}=1$ for $i=j$ and 0 for $i \neq j$.

While propagating from the source surface to the observer in a moving medium, the sound waves are convected by the mean flow velocity. Based on the Garrick triangle [20], the geometric interpretation for a source in a uniform rectilinear subsonic motion is represented in Fig. 9. The mean flow velocity is in the positive $x_{1}$-direction, i.e. $\mathbf{u}_{0}=$ $\left(U_{0}, 0,0\right)$. The source surface is described by $\left(\mathbf{y}, \tau_{e}\right)$ with $\mathbf{y}=\left(y_{1}, y_{2}, y_{3}\right)$ and the observer is described by $(\mathbf{x}, t)$ with $\mathbf{x}=\left(x_{1}, x_{2}, x_{3}\right)$. The moving medium is assumed to be homogeneous outside the source volume and its Mach number is $M_{0}$. 

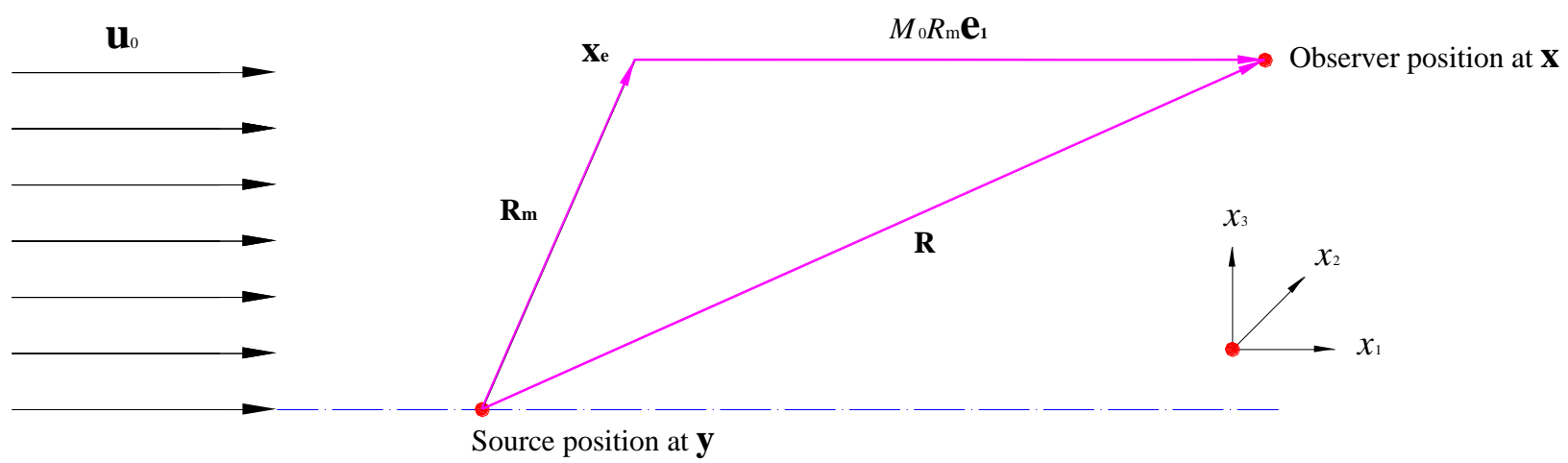

Fig. 9. Geometric interpretation of sound emission in a moving medium

Therefore, the radiation distance from the surface point to the observer is

$$
\mathbf{R}=\mathbf{x}-\mathbf{y}=\left(x_{1}-y_{1}\right) \mathbf{e}_{1}+\left(x_{2}-y_{2}\right) \mathbf{e}_{2}+\left(x_{3}-y_{3}\right) \mathbf{e}_{3},
$$

and the emission vector is presented by the following form,

$$
\mathbf{R}_{\mathrm{m}}=\mathbf{R}-\Delta t \mathbf{u}_{0}=\left(x_{1}-y_{1}-M_{0} R_{\mathrm{m}}\right) \mathbf{e}_{1}+\left(x_{2}-y_{2}\right) \mathbf{e}_{2}+\left(x_{3}-y_{3}\right) \mathbf{e}_{3},
$$

in which

$$
\Delta t \mathbf{u}_{0}=\left(M_{0} R_{\mathrm{m}}\right) \mathbf{e}_{\mathbf{1}} .
$$

The norm of the emission vector is

$$
\left|\mathbf{R}_{\mathrm{m}}\right|=R_{\mathrm{m}}=\sqrt{\left(x_{1}-y_{1}-M_{0} R_{\mathrm{m}}\right)^{2}+\left(x_{2}-y_{2}\right)^{2}+\left(x_{3}-y_{3}\right)^{2}} .
$$

Since the emission distance must be positive, the valid solution of Equation (8) is

$$
\begin{gathered}
R_{\mathrm{m}}=-\frac{M_{0}\left(x_{1}-y_{1}\right)}{1-M_{0}{ }^{2}} \\
+\sqrt{\left[\frac{M_{0}\left(x_{1}-y_{1}\right)}{1-M_{0}^{2}}\right]^{2}+\frac{\left(x_{1}-y_{1}\right)^{2}+\left(x_{2}-y_{2}\right)^{2}+\left(x_{3}-y_{3}\right)^{2}}{1-M_{0}{ }^{2}}} .
\end{gathered}
$$

It can be reformulated as

$$
R_{\mathrm{m}}=\frac{R^{*}-M_{0}\left(x_{1}-y_{1}\right)}{1-M_{0}^{2}},
$$

in which

$$
R^{*}=\sqrt{\left(x_{1}-y_{1}\right)^{2}+\left(1-M_{0}^{2}\right)\left[\left(x_{2}-y_{2}\right)^{2}+\left(x_{3}-y_{3}\right)^{2}\right]} .
$$

The spatial derivatives of the emission distance $R^{*}$ and $R_{\mathrm{m}}$ are $\tilde{R}_{i}^{*}=\frac{\partial R^{*}}{\partial x_{i}}$ and $\tilde{R}_{i}=\frac{\partial R_{\mathrm{m}}}{\partial x_{i}}$, which are introduced as follows:

$$
\begin{gathered}
\tilde{R}_{1}^{*}=\frac{x_{1}-y_{1}}{R^{*}}, \quad \tilde{R}_{2}^{*}=\left(1-M_{0}^{2}\right) \frac{x_{2}-y_{2}}{R^{*}}, \quad \tilde{R}_{3}^{*}=\left(1-M_{0}{ }^{2}\right) \frac{x_{3}-y_{3}}{R^{*}}, \\
\tilde{R}_{1}=\frac{1}{1-M_{0}{ }^{2}}\left(-M_{0}+\tilde{R}_{1}^{*}\right), \quad \tilde{R}_{2}=\frac{x_{2}-y_{2}}{R^{*}}, \quad \tilde{R}_{3}=\frac{x_{3}-y_{3}}{R^{*}} .
\end{gathered}
$$


Therefore, the thickness and loading noise source terms can be given as

$$
\begin{gathered}
4 \pi p_{\mathrm{T}}^{\prime}=\int_{f=0}\left[\left(1-M_{0} \tilde{R}_{1}\right) \frac{\dot{Q}_{i} n_{i}}{R^{*}}-U_{0} \frac{\tilde{R}_{1}^{*} Q_{i} n_{i}}{R^{* 2}}\right]_{\tau_{e}} d S(\boldsymbol{\eta}), \\
4 \pi p_{\mathrm{L}}^{\prime}=\int_{f=0}\left[\frac{1}{c_{0}} \frac{\dot{L}_{i j} n_{j} \tilde{R}_{i}}{R^{*}}+\frac{L_{i j} n_{j} \tilde{R}_{i}^{*}}{R^{* 2}}\right]_{\tau_{e}} d S(\boldsymbol{\eta}),
\end{gathered}
$$

in which $p_{\mathrm{T}}^{\prime}$ and $p_{\mathrm{L}}^{\prime}$ represent the thickness and loading noise contributions to the far-field sound pressure; a dot over a variable implies temporal derivatives with respect to the source time.

The time-dependent flow field on each panel (surface element) of the $\mathrm{FW}-\mathrm{H}$ integration surface and the corresponding geometry information are loaded from the computational fluid dynamics (CFD) simulation data. The integration surfaces used in the FW-H method to determine the noise contributions can be the solid surfaces of the geometries or permeable data surfaces surrounding the solid objects. A first-order scheme is applied for the spatial integration over the surface elements. The source time derivative is performed using fourth-order central differences. The advanced time approach is utilized for the numerical implementation [21]. At each flow-field time step and for each source element, the time (advanced time) at which the corresponding disturbance will reach the observer is calculated. Thus, the acoustic signals are finally built up in the observer time frame through a summation over all the contributions computed from the integration source domain. The sound pressure history at the observers is then constructed based on the calculation evolved along the source time axis.

\subsection{Numerical verification}

First, the far-field noise radiated from a point monopole and a point dipole source is predicted to validate the noise prediction code based on the convective $\mathrm{FW}-\mathrm{H}$ formulation. The harmonic sound fields of a point monopole and a point dipole in a moving medium are given respectively in terms of velocity potential as

$$
\phi(\mathbf{x}, t)=\frac{A}{4 \pi R^{*}} \exp \left[i \omega\left(t-\frac{R}{c_{0}}\right)\right],
$$

and

$$
\phi(\mathbf{x}, t)=\frac{\partial}{\partial x_{3}}\left\{\frac{A}{4 \pi R^{*}} \exp \left[i \omega\left(t-\frac{R}{c_{0}}\right)\right]\right\},
$$


where $R$ is the emission distance and $R^{*}$ is the radiation distance [19]; the nondimensional angular frequency is chosen as $\omega l / c_{0}=4 \pi / 46$ and the normalised amplitude is $A /\left(l c_{0}\right)=0.1$, where $l$ is a reference length. The source coordinate system is $\left(x_{1}, x_{2}, x_{3}\right)$. The dipole axis is aligned with the positive $x_{3}$-direction and transverse to the mean flow direction.

Assuming a mean flow $U_{0}$ is along the positive $x_{1}$-direction, the induced acoustic pressure, particle velocity and density fluctuation are

$$
\begin{gathered}
p^{\prime}(\mathbf{x}, t)=-\rho_{0}\left(\frac{\partial}{\partial t}+U_{0} \frac{\partial}{\partial x_{1}}\right) \phi, \\
u(\mathbf{x}, t)=\nabla \phi(\mathbf{x}, t), \\
\rho^{\prime}(\mathbf{x}, t)=p^{\prime}(\mathbf{x}, t) / c_{0}^{2} .
\end{gathered}
$$

The real parts of the time-varying pressure $p^{\prime}(\mathbf{x}, t)$, velocity $u(\mathbf{x}, t)$ and density $\rho^{\prime}(\mathbf{x}, t)$ are evaluated analytically on the integration permeable surface and used as source terms. Then the far-field acoustic pressure is predicted by using the convective $\mathrm{FW}-\mathrm{H}$ equation and compared with direct analytical calculations. The integration surface is defined as a cube that extends from $-5 l$ to $5 l$ in all three coordinate directions and the source is located at the centre of the cube. Each cube surface consists of $50 \times 50$ evenly spaced integration panels.

Fig. 10 depicts the comparisons of the time history of sound pressure between the analytical solution and the numerical prediction for the monopole and dipole sources. The observer is located at $(-50 l, 0,0)$ for the monopole case and at $(0,0,50 l)$ for the dipole case. The sound pressure is non-dimensionalised by $\rho_{0} c_{0}^{2}$ and the time is nondimensionalised by $l / c_{0}$. The period of oscillation is therefore 23 non-dimensional time units. This shows that the pressure fluctuation induced by the monopole is larger in magnitude than that induced by the dipole as expected. Moreover, the directivity patterns of the sound radiation for the two cases are depicted in Fig. 11, where the receivers are distributed uniformly along a circumference with a radius of $50 \mathrm{l}$ at an interval of $5^{\circ}$ and the root-mean-square pressure $p_{r m s}$ is displayed. Note that the directivity pattern is directional to upstream of the sound source due to convective amplification caused by the moving medium. Based on the comparisons in Figs. 10 and 11, it is found that the time history and directivity pattern of the far-field sound pressure are in excellent 
agreement between the analytical solution and the numerical calculation for both monopole and dipole test cases.

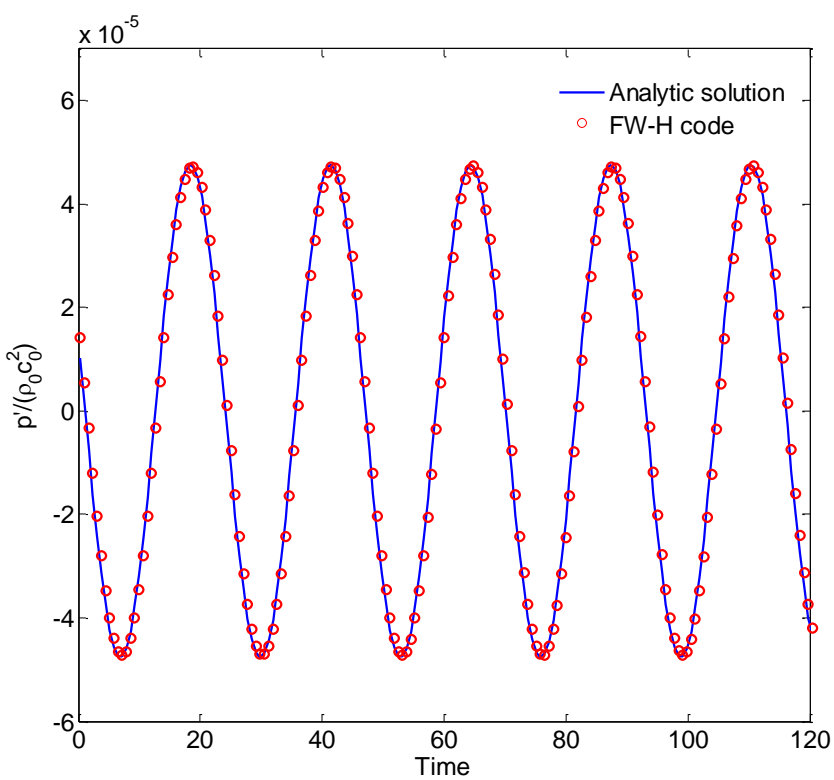

(a) Monopole source

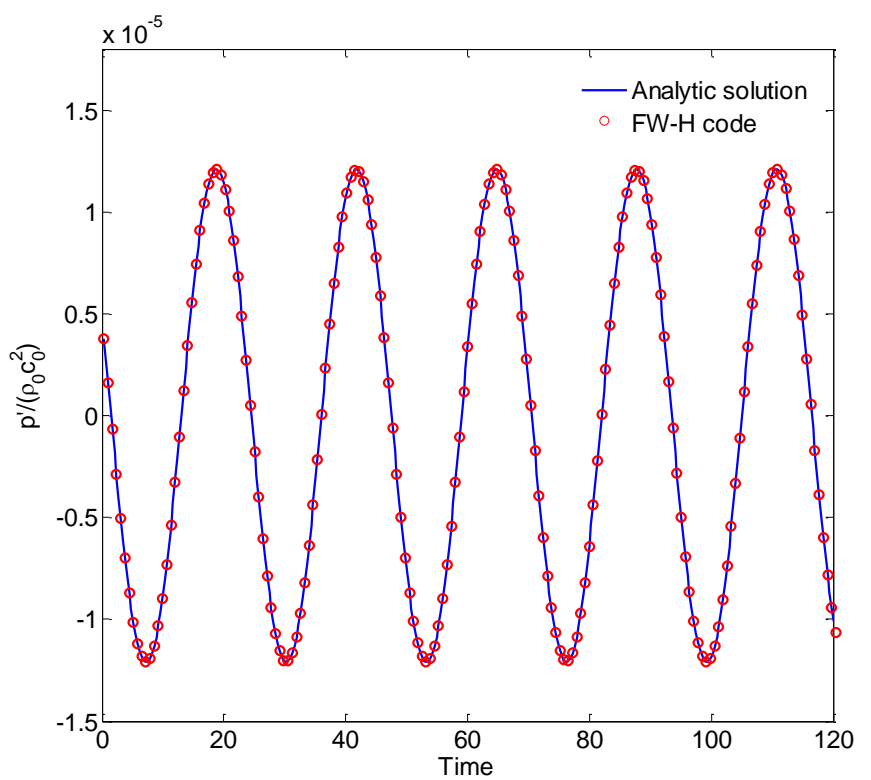

(b) Dipole source

Fig. 10. Comparisons of time history of the sound pressure between the analytic and predicted solutions

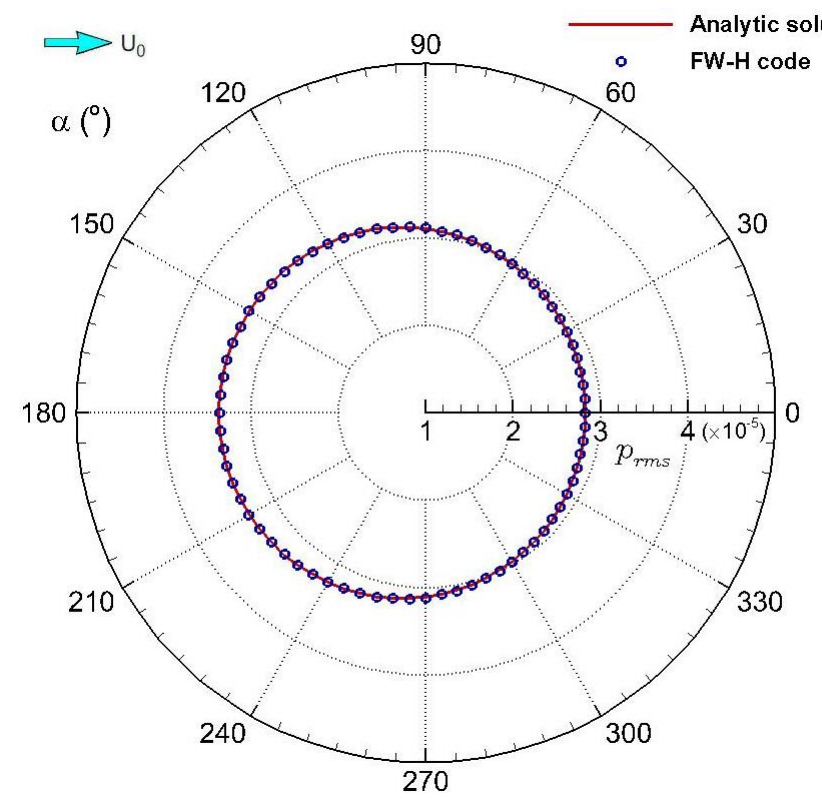

(a) Monopole source with the observer at $(-50 l, 0,0)$

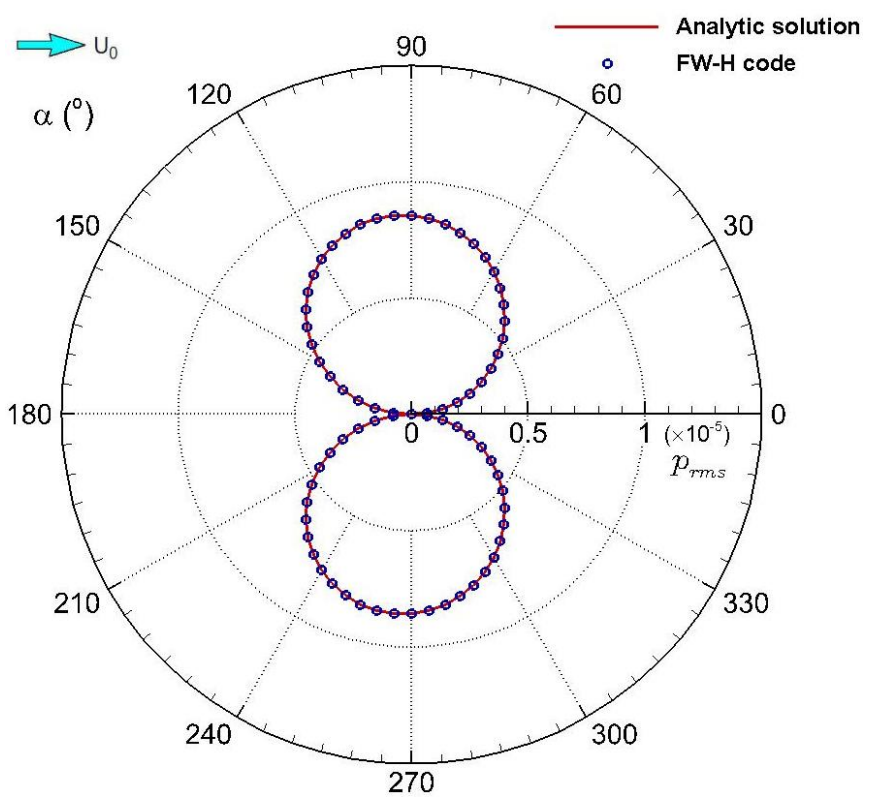

(b) Dipole source with the observer at $(0,0,50 l)$

Fig. 11. Comparisons of noise directivity between the analytic and predicted solutions 


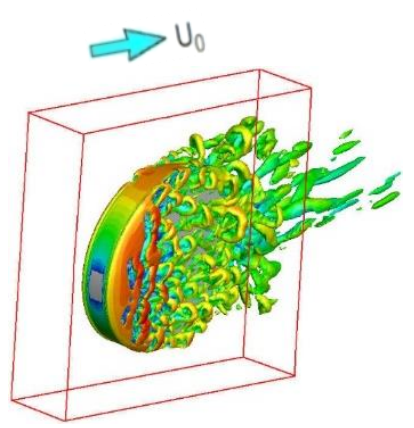

(a) Closed surface

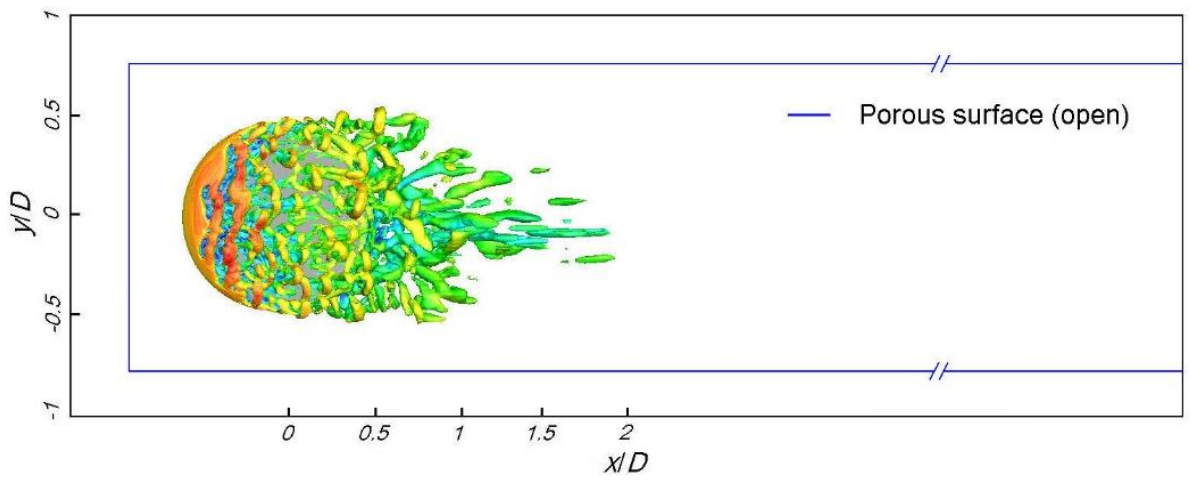

(b) Open surface

Fig. 12. Sketches of permeable surfaces used for the wheel test case

Next, an isolated wheel case (1:10 scale) is used to compare the noise predictions between the solid and permeable data surfaces. The wheel has a diameter $(D)$ of $92 \mathrm{~mm}$. The maximum cell size on the wheel surface is $0.98 \mathrm{~mm}$. The inlet velocity is $30 \mathrm{~m} / \mathrm{s}$ and the distance from the wheel to the nearest grid point is set as $10^{-5} \mathrm{~m}$, which yields a maximum value of $y^{+}$less than 1 . The DDES model is used for the numerical simulations.

In Fig. 12 sketches are shown of the wheel physical surfaces enclosed by the permeable surfaces. Also shown are the iso-surfaces of the second invariant of the velocity gradient $Q$ of the flow developed around the wheel. Fig. 12(a) displays the closed permeable surface constituting a porous box with dimensions of $1.5 D \times 1.5 D \times 0.5 D$ (length, height and width, where $D$ is the wheel diameter). By removing the downstream part of the closed permeable box and extending its top, bottom and side surfaces to the end of the computational domain, an open porous box with five permeable surfaces is established as shown in Fig. 12(b). The trackside receiver is located $2.5 \mathrm{~m}$ away laterally and $0.35 \mathrm{~m}$ above the wheel centre. The noise calculation using the physical solid surfaces is based on the classical FW-H method [16-18] and the current FW-H code is applied for the noise prediction on the permeable integration surfaces to compare the noise predictions from different methods.

It should be noted that in an incompressible flow solver, the wave speed is effectively infinite and the acoustic propagating waves cannot be properly captured by the permeable surfaces. However, these noise signals are relatively small for the cases at low Mach numbers. For example, based on the acoustic analogy method using the incompressible flow field solutions as the noise source data, Orselli et al. [22] found that 
the noise predicted using an off-body permeable surface was slightly higher than that calculated using the cylinder wall surfaces for the case of flow passing over a circular cylinder at a Mach number of 0.2 .

Fig. 13 displays the spectra of the noise radiated from the solid and two types of permeable surfaces. Figs. 13(a) and 13(b) show that the noise predictions from the solid and closed permeable surfaces agree well, especially in the frequency range below 900 $\mathrm{Hz}$. The differences at higher frequencies can be attributed to pseudo-sound that is generated by the turbulent eddies in the wheel wake region passing through the porous surfaces, as seen in Fig. 12(a) for the downstream outflow surface. Thus, compared with the results from the solid surfaces, the overall sound pressure level (OASPL) of noise predicted from the closed permeable surface is about $3.5 \mathrm{~dB}$ higher within the frequency range below $5 \mathrm{kHz}$. Keeping all the permeable surfaces further away from the geometries can reduce the pseudo-sound produced from the hydrodynamic perturbations; however, it will increase the computation cost greatly as the grids between the solid bodies and the permeable surfaces should be kept fine enough to get physically correct results [18].

Fig. 13(b) shows that the noise predicted from the open permeable surface corresponds fairly well with that from the solid and closed permeable surfaces in most of the frequency range. However, the noise level is about $20 \mathrm{~dB}$ higher below $200 \mathrm{~Hz}$, leading to unphysical results within this low-frequency range. This occurs because the $\mathrm{FW}-\mathrm{H}$ formulation uses Gauss's theorem to consider contributions from volume sources enclosed by the porous surfaces, which by definition should be closed. In the noise predictions using the open surfaces, contributions from the downstream surface are missing and make the summation of acoustic signals unbalanced there, especially at low frequencies. Similar influences of the open control surface at low frequencies were also observed in the noise predictions of a simplified landing gear using the FW-H method by Spalart et al. [23].

Consequently, based on the above validation test cases, it is demonstrated that the FW$\mathrm{H}$ code can be used for far-field noise prediction. However, it is seen that when the open surfaces are used for noise predictions, errors are introduced by the missing part of the surface at low frequencies. 


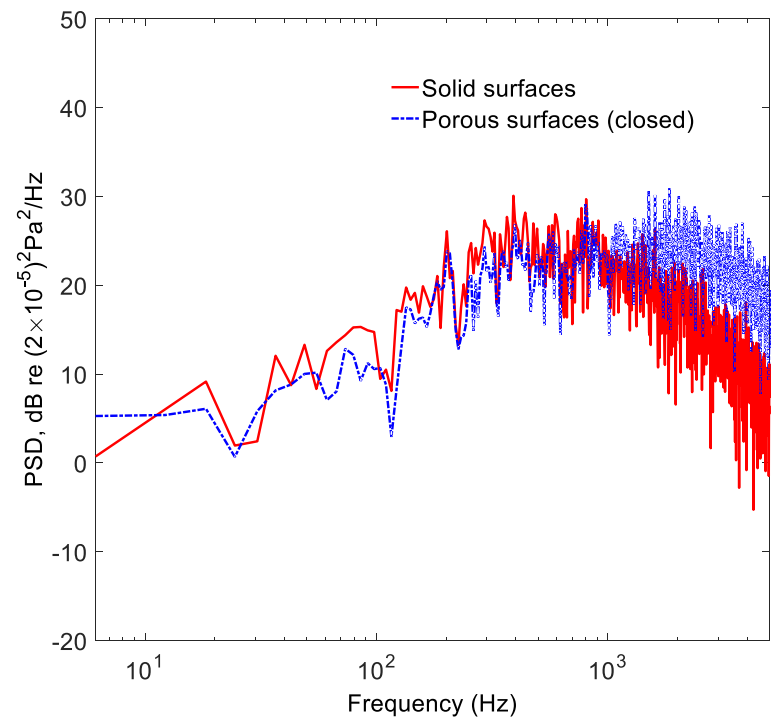

(a) Solid and closed permeable surfaces (narrow-band frequency resolution)

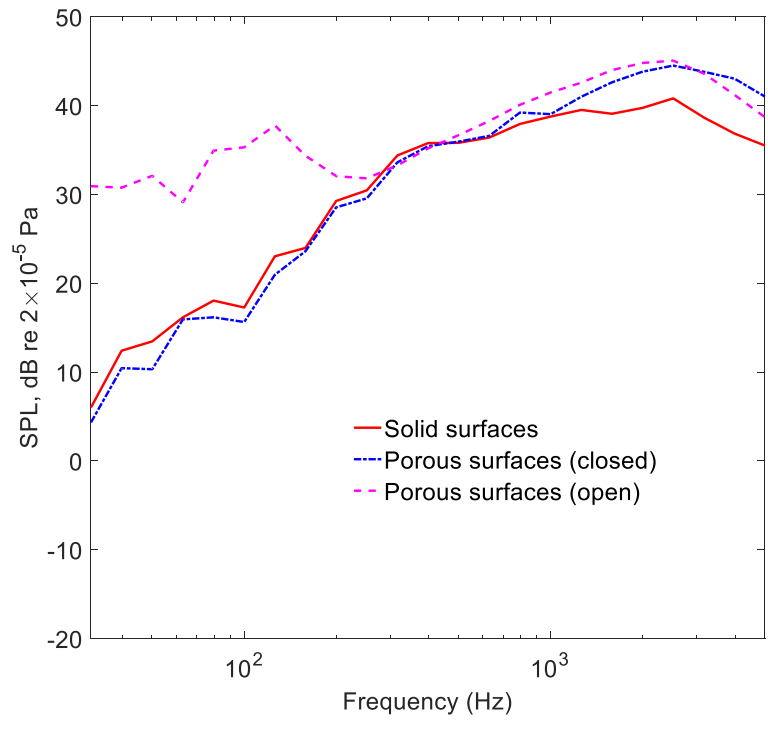

(b) Solid and permeable surfaces ( $1 / 3$ octave spectra)

Fig. 13. Comparisons of spectra of the far-field sound pressure

\section{Influence of Bogie Cavity and Fairing on Sound Generation}

When the transient flow field has become statistically steady, the far-field noise can be predicted by the FW-H method based on the near-field unsteady flow data. The CFD simulations were run for $1.1 \mathrm{~s}$ corresponding to 17 times the flow-through time for both cases and the length of the time signal used as input for noise calculation corresponds to the last $0.52 \mathrm{~s}$ of the computations. The power spectral density is computed from the predicted far-field noise time history by Welch's method and averaged over 50\% overlapping segments using a Hanning window applied to five segments, giving a frequency resolution of $6 \mathrm{~Hz}$. The OASPL is calculated from the PSD within a certain frequency range, as explained below.

Fig. 14 displays the spectra of the radiated noise from the half bogie symmetrical along the axle mid-span at the receiver located at $(0,2.5 \mathrm{~m}, 0)$ with the origin at the bogie centre. The noise calculations are based on the solid integration surfaces. It is found that compared to the isolated bogie case [9], the noise level from the bogie-inside-cavity case without the fairing is significantly reduced (the OASPL below $2 \mathrm{kHz}$ changes from 72.7 $\mathrm{dB}$ to $55.2 \mathrm{~dB}$ ) as the incident flow velocity becomes smaller and no massive vortex shedding is generated around the bogie located inside the cavity. 


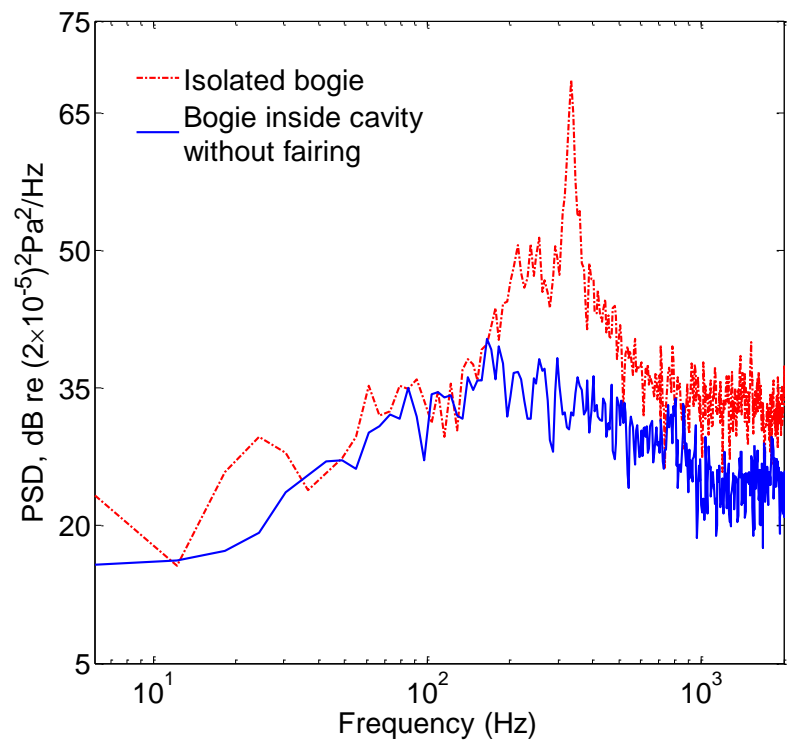

Fig. 14. Comparisons of spectra of acoustic pressure on far-field receiver based on solid integration surfaces for isolated bogie [9] and bogie inside the cavity without fairing

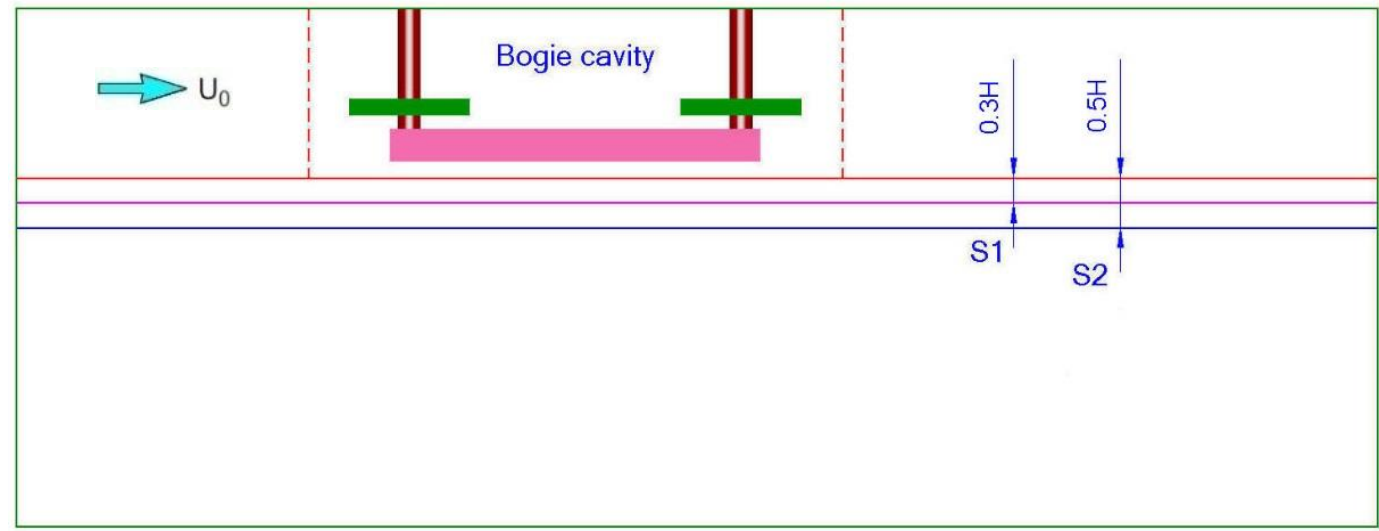

Fig. 15. Sketch of porous surfaces used for FW-H integration (top view, not to scale)

Due to the complexity of the geometry of the bogie-inside-cavity cases, the FW-H method based on the solid surfaces has the disadvantage that it does not properly represent the sound radiation from hidden surfaces. Therefore, for the cases without and with the fairing, the radiated noise is calculated using the acoustic analogy implemented on a permeable surface located at a distance from the carbody sidewall, extending over the whole height and length of the domain. Fig. 15 illustrates the porous surfaces (S1 and S2) used for the $\mathrm{FW}-\mathrm{H}$ integration. The gap between the porous surface (S1) and the carbody side wall is $0.3 H=21 \mathrm{~mm}$ (where $H$ is the bogie cavity height of $70 \mathrm{~mm}$ ) and that for the porous surface (S2) is $0.5 H=35 \mathrm{~mm}$. The mesh between the two permeable surfaces is kept fine enough to ensure that the flow calculations with high resolution are performed around 
these data surfaces. In the current calculations, there are 129,666 panels on the permeable data surface for the case without the fairing and 128,010 panels for the case with the fairing.

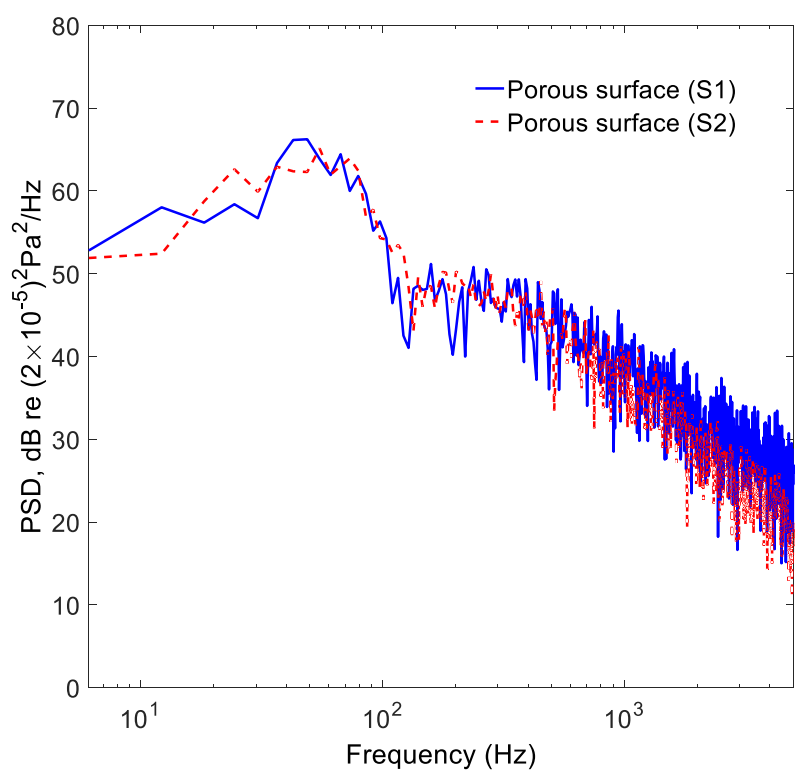

(a) Two porous surfaces

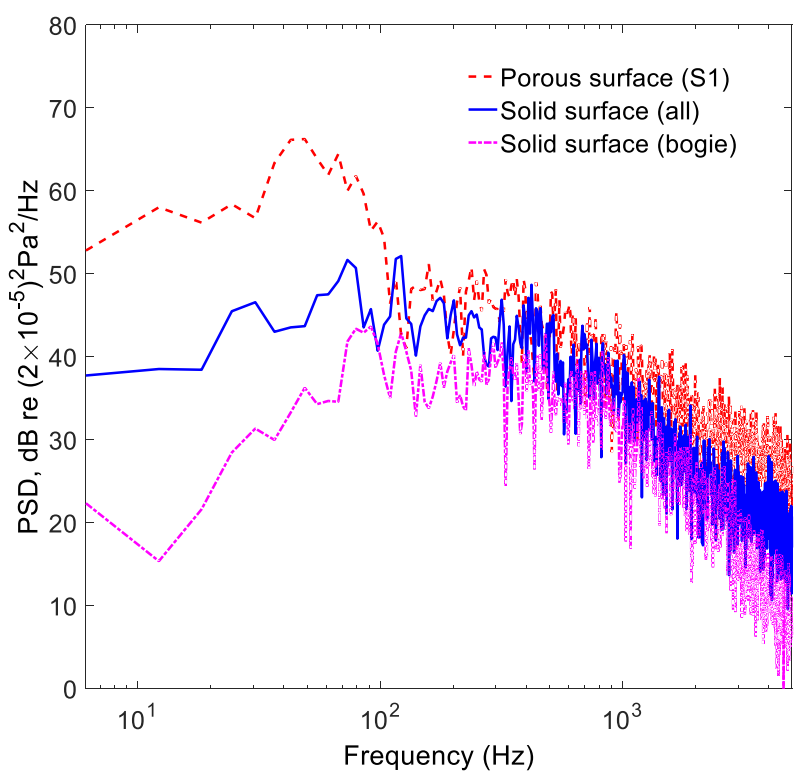

(b) Porous and solid surfaces

Fig. 16. Comparisons of far-field noise spectra in the bogie cavity case without the fairing

Fig. 16 displays the comparisons of the far-field noise spectra of the case without the fairing based on the numerical simulations on the symmetrical half bogie (shown in Fig. 15) using the permeable data surfaces in the $\mathrm{FW}-\mathrm{H}$ method. The receiver is located at $2.5 \mathrm{~m}$ laterally and $0.35 \mathrm{~m}$ above the bogie centre. Fig. 16(a) shows that the noise predictions based on the two permeable surfaces (S1 and S2) are very close for frequencies up to $2 \mathrm{kHz}$, indicating that the influence of the placement of these two surfaces on the calculated far-field acoustic signals is negligible. Fig. 16(b) compares the noise predictions from permeable (S1) and solid data surfaces for the case without the fairing. This shows that the noise generated from all solid surfaces is about $6 \mathrm{~dB}$ higher than that from the bogie wall surfaces alone in the frequency range below $2 \mathrm{kHz}$. This implies that the carbody, especially the bogie cavity rear wall and trailing edge, produce a large contribution of noise. Moreover, compared with the noise generated from all wall surfaces, the noise amplitude predicted by the permeable integration surfaces is much higher below $120 \mathrm{~Hz}$ due to the unphysical results obtained using the open permeable surface, as discussed earlier in the isolated wheel case. At high frequencies the pseudonoise generated at the permeable surface has a large effect on the results; however, due 
to its low level in this frequency region, it has negligible effect on the overall levels. Therefore, the frequency range between $120 \mathrm{~Hz}$ and $2 \mathrm{kHz}$ will be used for noise prediction. Within this frequency range, the noise generated from the permeable integration surface $(\mathrm{S} 1)$ is $3.1 \mathrm{~dB}$ higher than that from all solid surfaces due to the pseudo-sound generated by the turbulent eddies passing through the permeable surface; from the permeable surface $\mathrm{S} 2$ the difference is $2.6 \mathrm{~dB}$. The quadrupole noise contributions from outside the solid surface but inside the porous surface are accounted for by the surface integrals although they are rather small for current cases at low Mach numbers and therefore not expected to influence the results.

To reduce the computation cost, the permeable surface (S1) is used for the noise predictions of the subsequent cases of the bogie inside the cavity. For the case including the fairing, there is no surface shape discontinuity around the bogie area on the carbody side wall. Thus the flow would be less unsteady on the side permeable surface (S1) and the difference between the noise predictions on the two surfaces ( $\mathrm{S} 1$ and $\mathrm{S} 2$ ) is expected to be smaller compared with the case without the fairing.

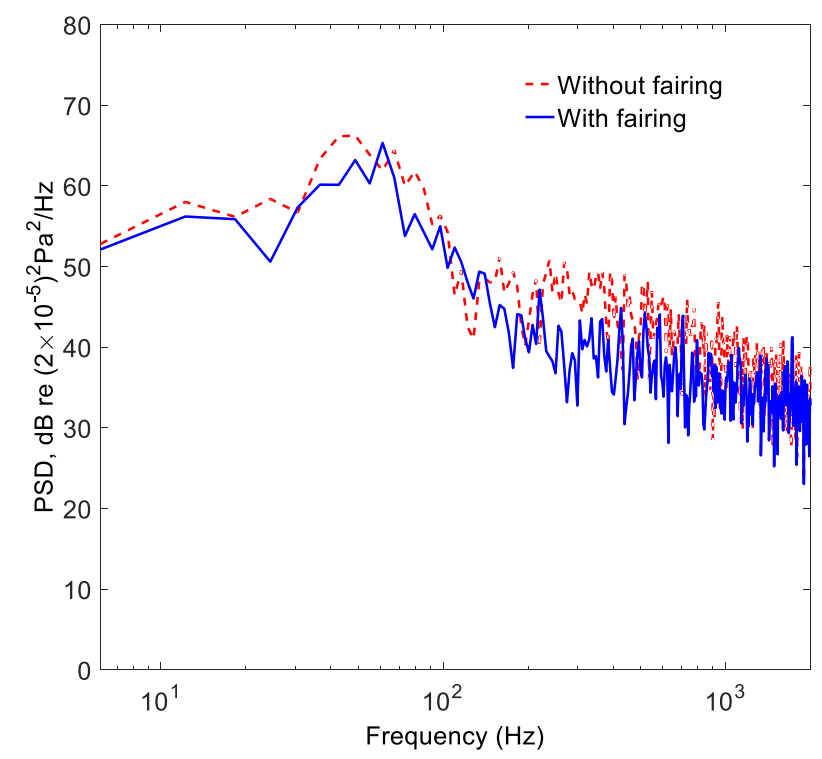

(a) Noise spectra

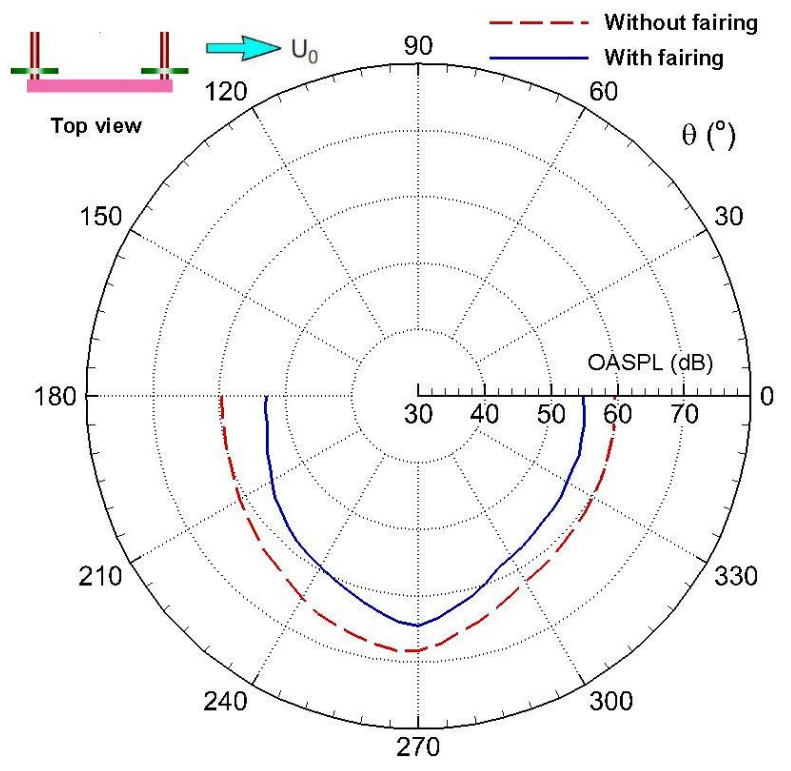

(b) Noise directivity

Fig. 17. Comparisons of far-field noise prediction from the permeable surface

Fig. 17 shows the comparisons of the far-field noise predictions from the permeable surface (S1) for the cases without and with the fairing. Fig. 17(a) depicts the far-field noise spectra of these two cases. The receiver is again located $2.5 \mathrm{~m}$ away and $0.35 \mathrm{~m}$ above 
the bogie centre. It is found that the level in the frequency range between $120 \mathrm{~Hz}$ and 2 $\mathrm{kHz}$ is $4 \mathrm{~dB}$ lower for the bogie cavity case with the fairing than the case without the fairing. This is in contrast to the noise generated by the sources on the bogie surfaces which showed higher levels in the case with fairing (Fig. 8). This is because although the flow interaction within the bogie cavity covered by the fairing introduces additional unsteadiness on the bogie surfaces, the fairing is effective in limiting the flow unsteadiness developed within the bogie cavity and reducing the flow unsteadiness around the carbody outside the bogie cavity, and thus reducing the noise sources in those areas. Therefore, the bogie fairing is able to achieve an overall noise reduction in the farfield.

Fig. 17(b) displays the noise directivity calculated from the permeable surface for both cases. The OASPL is again calculated from the PSD over the frequency range from 120 $\mathrm{Hz}$ to $2 \mathrm{kHz}$ and the receivers are distributed uniformly around a semicircle with radius $2.5 \mathrm{~m}$ from the bogie centre in the bogie horizontal central plane. A lateral dipole-like pattern of noise directivity is predicted with noise amplitudes between 3.8 and $6.6 \mathrm{~dB}$ lower for the case with the fairing compared with the case without the fairing. This noise reduction is obtained due to the shielding of the flow unsteadiness within the bogie cavity by the fairing and the change of the hydrodynamic flow field outside the fairing. It does not take into account the scattering of sound waves by the solid surfaces, for which the compressible flow solutions would be required.

\section{Conclusions}

The flow behaviour and the aerodynamic noise characteristics of a simplified bogie located inside a bogie cavity with and without a fairing have been studied using the DDES model and the FW-H acoustic analogy. Results show that a highly unsteady flow is generated inside the bogie cavity due to the considerably strong flow interactions occurring there and the flow within the bogie cavity is characterized by vortex shedding, turbulent wake/vortical-structure and wake/wake interactions. The surface shape discontinuity of the bogie cavity along the carbody side walls in the case without the fairing generates a strong unsteady flow around these regions. When the bogie cavity is 
shielded by a fairing, the flow interactions are limited inside the bogie cavity and its development outside the fairing is weakened greatly.

Additionally, the noise radiated to the trackside is predicted using a permeable integration surface close to the bogie and parallel to the carbody side wall. It is found that the flow separation and the vortex shedding around the geometries are the main contributors to the aerodynamic noise generation in the current cases. The bogie fairing is effective in reducing the noise levels generated in most of the frequency range and a noise reduction about $5 \mathrm{~dB}$ is achieved in the farfield for the current model case due to the influence of the fairing in shielding the flow unsteadiness within the bogie cavity and reducing the flow fluctuations outside the fairing.

Note that for a full-scale bogie case in reality, the Reynolds number will be much higher than in the scale model case considered, and the aerodynamic and aeroacoustic behaviour will be different for some parts. Moreover, the presence of turbulent inflow and more detailed geometries including the ground underneath will lead to more complex flow structures, which will affect the noise generation and radiation. Nevertheless, these findings from the scale models are useful in understanding the corresponding effects on an actual bogie fairing mounted on a high-speed train bogie.

\section{Acknowledgements}

The numerical simulations were performed on the Iridis4 cluster managed by the University of Southampton. The first author is grateful for the funding provided by the University of Southampton and the support from Shanghai Key Lab of Vehicle Aerodynamics and Vehicle Thermal Management Systems. The data supporting this study are openly available from the University of Southampton repository at http://dx.doi.org/10.5258/SOTON/xxxxx. 


\section{References}

[1] D.J. Thompson, E. Latorre Iglesias, X.W. Liu, J.Y. Zhu, Z.W. Hu. Recent developments in the prediction and control of aerodynamic noise from highspeed trains. International Journal of Rail Transportation, 2015, 3(3): 119-150.

[2] D.J. Thompson. Railway noise and vibration: mechanisms, modelling and means of control. Elsevier, Oxford, UK, 2008.

[3] C. Mellet, F. Létourneaux, F. Poisson, C. Talotte. High speed train noise emission: Latest investigation of the aerodynamic/rolling noise contribution. Journal of Sound and Vibration, 2006, 293: 535-546.

[4] K. Nagakura. Localization of aerodynamic noise sources of Shinkansen trains. Journal of Sound and Vibration, 2006, 293: 547-556.

[5] Hee-Min Noh, Sunghoon Choi, Sukyoon Hong, Seog-Won Kim. Investigation of noise sources in high-speed trains. Proceedings of the Institution of Mechanical Engineers Part F: Journal of Rail and Rapid Transit, 2014, 228(3): 307-322.

[6] E. Latorre Iglesias, D.J. Thompson, M.G. Smith, T. Kitagawa, N. Yamazaki. Anechoic wind tunnel tests on high-speed train. Proc. 7th Forum Acusticum 2014, Krakow, Poland.

[7] N. Frémion, N. Vincent, M. Jacob, G. Robert, A. Louisot, S. Guerrand. Aerodynamic noise radiated by the intercoach spacing and the bogie of a highspeed train. Journal of Sound and Vibration, 2000, 231(3): 577-593.

[8] J.Y. Zhu, Z.W. Hu, D.J. Thompson. Flow simulation and aerodynamic noise prediction for a high-speed train wheelset. International Journal of Aeroacoustics, 2014, 13(7\&8): 533-552.

[9] J.Y. Zhu, Z.W. Hu, D.J. Thompson. Flow behaviour and aeroacoustic characteristics of a simplified high-speed train bogie. Proceedings of the Institution of Mechanical Engineers Part F: Journal of Rail and Rapid Transit, Published online September 30, 2015.

[10] B. Schulte-Werning. Research of European railway operators to reduce the environmental impact of high-speed trains. Proceedings of the Institution of Mechanical Engineers Part F: Journal of Rail and Rapid Transit, 2003, 217(4): 249257. 
[11] A. Torii, J. Ito. Development of the series 700 Shinkansen train-set, improvement of noise level. Proceedings of World Congress on Railway Research 1999, Tokyo, Japan, 1999.

[12] J.Y. Zhu. Aerodynamic Noise of High-speed Train Bogies. PhD Thesis. University of Southampton, 2015.

[13] M. Wang, J.B. Freund, S.K. Lele. Computational prediction of flow-generated sound. Annual Review of Fluid Mechanics, 2006, 38: 483-512.

[14] J. Ask, L. Davidson. An acoustic analogy applied to the laminar upstream flow over an open 2D cavity. Comptes Rendus Mecanique, 2005, 333: 660-665.

[15] P.R. Spalart, S. Deck, M.L. Shur, K.D. Squires, M.Kh. Strelets, A.K. Travin. A new version of detached-eddy simulation, resistant to ambiguous grid densities. Theoretical and Computational Fluid Dynamics, 2006, 20: 181-195.

[16] J.E. Ffowcs-Williams, D.L. Hawkings. Sound radiation from turbulence and surfaces in arbitrary motion. Philosophical Transactions of the Royal Society of London, 1969, 342: 264-321.

[17] F. Farassat. Derivation of Formulations 1 and 1 A of Farassat. NASA/TM-214853, 2007.

[18] K.S. Brentner, F. Farassat. Modelling aerodynamically generated sound of helicopter rotors. Progress in Aerospace Sciences, 2003, 39: 83-120.

[19] A. Najafi-Yazdi, G.A. Brès, L. Mongeau. An acoustic analogy formulation for moving sources in uniformly moving media. Proceedings of the Royal Society of London, Series A, 2011, 467: 144-165.

[20] I.E. Garrick, E.W. Watkins. A theoretical study of the effect of forward speed on the free-space sound-pressure field around propellers. NASA/TM-79844, 1954.

[21] D. Casalino. An advanced time approach for acoustic analogy predictions. Journal of Sound and Vibration, 2003, 261: 583-612.

[22] R.M. Orselli, J.R. Meneghini, F. Saltara. Two and three-dimensional simulation of sound generated by flow around a circular cylinder. AIAA Paper 2009-3270, 2009.

[23] P.R. Spalart, M.L. Shur, M.Kh. Strelets, A.K. Travin. Initial noise predictions for rudimentary landing gear. Journal of Sound and Vibration, 2011, 330, 4180-4195. 